\title{
On Common-Sense Justifications of Optimality-Theoretic Constraints
}

\author{
Gereon Müller
}

Universităt Tübingen, SfS, Wilhelmstr. 113, 72074 Tübingen gereon.mueller@uni-tuebingen.de

\$1 Haspelmath notes that optimality-theoretic constraints that have been proposed in the literature often seem to lend themselves to a functional explanation; sometimes, they are in fact explicitly justified by invoking functional notions. He correctly points out that "strictly speaking, [functional] justifications are irrelevant in a theory that assumes innate constraints" (\$2), as is the case in standard optimality theory. ${ }^{1}$ Haspelmath concludes from this that grammatical constraints result directly from functional constraints on language users, via a process of diachronic adaptation. A different conclusion must be drawn if a direct functional motivation of optimality-theoretic constraints turns out to be neither necessary nor possible. On this view, grammar is autonomous, and a constraint qualifies as "good" if it meets the requirement of theoryinternal elegance. This concept includes generality of application and simplicity of formulation (e.g., avoidance of Boolean operations in constraint definitions), but not functional, common-sense justification. I will try to defend this latter view here, based on evidence from syntax.

\$2 First, the user constraints that Haspelmath postulates as functional motivations for optimality-theoretic constraints are typically very different in formulation and scope. This holds, e.g., for Grimshaw's (1997) STAY and Haspelmath's corresponding user constraint: The former prohibits any instance of movement, and is violated regularly in well-formed sentences; the latter blocks non-canonical linearization that creates potential ambiguity (not movement per se), and is thus violated less often. Clearly, the two constraints make different predictions: Both are (fatally) violated in examples involving multiple wh-movement in English ( ${ }^{*} I$ wonder who what gave 10 Mary). However, whereas STAY also successfully rules out the same structure in German, the correspon-

1 This clear statement of the "pseudo-functionalist fallacy" that seems to be fairly widespread among (non-functionalist) grammarians (and perhaps particularly those working in optimality theory) is spot-on, and should be taken to heart, independently of what one thinks of Haspelmath's own proposal: There is no room for functional, common-sense motivations of constraints that one believes to be innate. 
ding user constraint does not: Because of explicit Case morphology, there is no ambiguity (*I frage mich, wer wem (daß) ihn vorgestellt hat). Moreover, we expect that string-vacuous movement that does not create non-canonical linearization does not violate user-optimal STAY, and this would fail to exclude string-vacuous raising of the wh-subject and the auxiliary in subject-initial questions in English ( $W h o_{1}$ will $t_{1} t_{2}$ leave?), in contrast to Grimshaw's original StaY. Finally, Haspelmath holds user-optimal STAY responsible for the absence of free word order in English (cf. his (15-ab)). Given rich Case morphology, free word order structures in languages like German, Old English, Russian, and Korean do not violate this functional constraint. Thus, Haspelmath accounts for variation with respect to free word order without recourse to different constraint rankings in syntax - middle field-internal violations of user-optimal STAY are assumed to be fatal. This then raises the question of why Bulgarian, which has an impoverished Case system that is very similar to that of English, exhibits free word order structures of roughly the Russian type (Molxova (1970), Rudin (1985)). Here, it looks as though the only solution might be to assume that violations of (user-optimal) STAY are not fatal in Buggarian after all - but if this is so, (user-optimal) STAY as such cannot be the reason for the absence of free word order structures in English.

Problems of this kind abound. E.g., the functional reconstruction of Grimshaw \& Samek-Lodovici's (1998) DroP TOPIC in effect makes this constraint inviolable: Languages with rich subject agreement respect DROP ToPIc by omitting a topical subject pronoun, and other languages respect it vacuously by not omitting a topical subject pronoun. This is not compatible with the use of Drop TOPIC in optimality-theoretic analyses, where this constraint is counterbalanced by a requirement to overtly realize subject pronouns - so English can violate DROP ToPIC to fulfill the latter requirement. ${ }^{2}$ Since discrepancies between standard optimality-theoretic constraints and their functional reconstructions oocur systematically, we face a dilemma: If a given grammatical constraint is directly equated with a postulated user constraint, the analyses which originally motivated the grammatical constraint must be abandoned; but if the grammatical constraint is merely viewed as the indirect consequence of a postulated user constraint that "underlies" it, there must be an additional, unexplained mechanism that translates user constraints into grammatical constraints.

$\$ 3$ Haspelmath states that "there is probably no need to go into the details of what exactly makes language structures 'good' for speakers and hearers, i.e.,

2 Similarly, the idea that Pesetsky's (1998) Teregraph might subsume DropTopic (\$6.3) is incompatible with actual optimality-theoretic analyses because TELEGRAPH forces PF-deletion of existing syntactic material, whereas DROPTOPIC forces the absence of syntactic material. 
what constitutes user optimality ... The most important cost factors are motor costs and cognitive processing costs, and the most important benefits are informativeness and persuasiveness" ( $(3)$. I am not convinced that a precise characterization of what counts as a functional explanation is unnecessary. In the absence of clear, independently verifiable criteria, functional explanations will often simply be common-sense justifications and thereby run the risk of being post hoc (it is usually not hard to contrive some functional motivation for almost any given constraint) and arbitrary (in the case of grammatical constraints that are rejected on functional grounds). Indeed, it turns out that psycholinguistic evidence may very well contradict our common-sense view regarding "processing costs." Consider again STAY. In its original formulation, this constraint blocks movement. A straightforward functional reconstruction would be to assume that each movement operation is costly for the parser. This, essentially, is the derivational theory of complexity of the 60 's, which was not strongly supported by psycholinguistic evidence. Another functional reconstruction is the one envisaged by Haspelmath: What is costly for the parser on this view is a non-canonical linearization of (Case-) ambiguous elements. However, this hypothesis is not confirmed by psycholinguistic experiments. Schlesewsky et al. (1997) show that locally unambiguous object-initial whquestions exhibit higher reading times than locally unambiguous subject-initial wh-questions; i.e., we find the same pattern as with locally ambiguous wh-questions. This suggests that what is costly for the parser is in fact path length (or non-minimal links), and whereas such notions may indeed play a role in grammatical theory (cf. Chomsky (1995), and Fanselow (1999) for a direct application), it is shown in Müller (to appear b) that a version of STAY that is based on path length is fundamentally incompatible with the optimalitytheoretic analyses given in Grimshaw (1997), Müller (1997 b), Costa (1998), Legendre et al. (1998), Vikner (to appear), and elsewhere. Again, the problem is more general: There will often be incompatibilities of common-sense justifications and the actual psycholinguistic evidence.

$\$ 4$ Despite Haspelmath's claim that "most of the widely used, non-ephemeral constraints can be reformulated in user-optimality terms" ( $\$ 3)$, there are many cases where this is far from obvious. This holds for the whole class of (left- and right-) alignment constraints in syntax that have been taken over from phonology. Such constraints are already present in Grimshaw (1997) and Pesetsky (1998), and they have come to play a crucial role in much recent work (cf. Costa (1998), Legendre (1998), Samek-Lodovici (1998)). ${ }^{3}$ The same goes for

3 The complex version of Pesetsky's complementizer alignment constraint $L E(C P)$ that is criticized by Haspelmath as a theoretical construct may also fail to meet the requirement of theory-internal generality and simplicity, but this does not hold for the simple version that suffices for most of the data. 
Grimshaw's (1997) Ов-HD that requires the presence of visible heads in projections; for Legendre et al.'s (1998) constraints on absorption and adjunction that regulate wh-dependencies; for the binding-theoretic constraints in Wilson (to appear), the Case and Person constraints in Woolford (to appear) and Aissen (to appear), respectively (one may claim that 1./2.Pers. is treated differently from 3 . Pers. in functional terms, but this does not yet explain why there are constraints against both); and so on. An important class of constraints that lack obvious user constraint counterparts are markedness constraints that trigger displacement. A constraint like the Wh-Crrerion (Grimshaw (1997), Müller (1997 b), Legendre et al. (1998), Ackema \& Neeleman (1998)) forces wh-phrases to be in SpecC. Over the years, a substantial body of evidence has been accumulated that argues against a semantic/functional motivation of wh-movement, and for a purely formal, grammar-internal motivation: Whphrases are not necessarily operators (Pesetsky (1987), Berman (1991)); some cases of wh-movement must partly be undone semantically (Beck (1996)); all wh-phrases can in principle be interpreted in situ (Groenendijk \& Stokhof (1982)); wh-phrases may respect the WH-CRrTERION by undergoing partial movement to an embedded non-scope position (van Riemsdijk (1982)), etc. A SUDECT constraint (Grimshaw (1997), Grimshaw \& Samek-Lodovici (1998), Costa (1998)) forces subjects to be in Specl, with no grammar-external motivation in sight. A V/2-CoNsTraINT triggers filling of left-peripheral specifiers in $V / 2$ languages, without any functional restriction on this position: $\mathrm{SpecV} / 2$ can be interpreted as topic, as contrastive or completive focus, via reconstruction, or not at all (expletive insertion). Even the PrONOUN-Crrterion (Müller (to appear a)), which forces unstressed pronouns to be in the Wackernagel position (Ich habe (es) gestern dem Fritz ("es) gegeben), does not necessarily provide evidence for a functional motivation. At first sight, the constraint appears to be derivable from Behagel's Gesetz der wachsenden Glieder, which Hawkins (1990) considers a parsing strategy (also cf. Primus (1994)). However, there is psycholinguistic evidence both in favour of such a principle (cf. Hawkins (1990) and references cited there), and against it (cf. McDonald et al. (1993), a study that minimizes intervening factors).

$\$ 5$ So far, I haven't said anything about the model of diachronic adaptation. If the considerations in $\$ 2-4$ are on the right track, the issue of direct adaptation does not arise because grammatical constraints cannot directly be based on user constraints (and many grammatical constraints cannot have a functional basis at all). This leaves as possibilities indirect adaptation of a subset of grammatical constraints, and complete independence. At present $I$ take it to be an open question whether an indirect influence of user constraints on some grammatical constraints does exist. For the sake of argument, suppose that it does (perhaps in the case of ANIM > INANIM, assuming that a functional counterpart can be 
motivated independently of intuitive plausibility). ${ }^{4}$ Then, diachronic adaptation may indeed be a better model than biological adaptation (it evades the teleological fallacy and is less speculative). On this view, an optimality-theoretic grammar would consist of a core of innate constraints without functional motivation, and a periphery of constraints with a functional basis, with both constraint types interacting through violability and ranking. Needless to say, though, this is pure speculation; it may just as well be the case that no grammatical constraint has any functional basis. ${ }^{5}$ Given the lack of decisive evidence, my conclusion is that, for the time being, the (generative) grammarian is well advised to concentrate on how questions, and postpone why questions until our understanding of cognitive issues has deepened, and these questions can be answered (or even asked) properly.

4 In contrast, RECOVERABn ITY seems more like a meta-constraint on grammars than like a genuine grammatical constraint; so the question of a functional justification does not arise. Compare Chomsky's (1965) statement that this constraint "need never be stated in the grammar, since it is a condition on the functioning of grammars" (p. 255), and Pesetsky's (1998) assumption that, unlike other optimality-theoretic constraints, RECOVERABRITY can never be violated by a well-formed sentence.

5 This would not preclude looking for "optimal design specification" (Chomsky (1998)) in grammar, as long as the goal is maximal generality and simplicity, and not maximal reduction to functional considerations. 